\title{
Experimental study of the evaporation of sessile nanoliter liquid drops
}

\author{
Andrey Semenov ${ }^{1,2^{*}}$ and Dmitry Zaitsev ${ }^{1,2}$ \\ ${ }^{1}$ Kutateladze Institute of Thermophysics, 630090, Lavrentiev ave. 1, Novosibirsk, Russia \\ ${ }^{2}$ Novosibirsk State University, 630090, Pirogova str. 2, Novosibirsk, Russia
}

\begin{abstract}
Evaporation of a sessile nanoliter water droplet was investigated experimentally at a temperature difference between the solid substrate and surrounding atmosphere from 30 to $50{ }^{\circ} \mathrm{C}$. The studies were performed on the float glass substrate with aluminum nanocoating of optical quality. The research has shown that the specific rate of evaporation (mass loss per unit of the drop surface area) increases with the decrease in droplet volume and at the last stage several times exceeds the initial value.
\end{abstract}

\section{Introduction}

The phenomenon of liquid drop evaporation, which takes place in a variety of technological systems in power engineering, agriculture, medicine, cooling systems, chemical and other industries [1], has been actively investigated during the last few years [2-5]. In most experimental and theoretical studies found in the literature, isothermal evaporation of water droplets has been studied. Publications on nonisothermal evaporation of liquid droplets with a sufficiently high temperature difference between the solid surface and the atmosphere are very limited.

In this paper, we study the process of evaporation of sessile liquid droplet with initial volume less than $1 \mu \mathrm{l}$. A drop of liquid is placed on a surface with controlled wettability. Then the liquid drop is heated until quasi-stationary heat transfer between the solid substrate and the drop. Distilled deionized nano-filtered water of Milli-Q company is used as the working fluid. The use of water with a relatively high boiling point as working fluid provides for a rather detailed investigation of the influence of temperature difference between the solid surface and the surrounding atmosphere on the rate of evaporation and heat transfer. The temperature difference between the solid and external atmosphere varies from 30 to $50{ }^{\circ} \mathrm{C}$.

\section{Apparatus and technics}

The experiments on water drops evaporation in the open atmosphere were made on the experimental stand the photograph of which is presented in Fig. 1. The work area was an

\footnotetext{
* Corresponding author: semenov.itp@gmail.com
} 
aluminum plate of $15 \mathrm{~mm}$ thickness, on whose bottom side the heater was located. The used heater was a Peltier element with dimensions $40 \times 40 \mathrm{~mm}^{2}$. On the upper side of the aluminum plate the investigated substrate was fixed. The substrate is a float glass disk (with diameter of $52 \mathrm{~mm}$ and thickness of $3.2 \mathrm{~mm}$ ) with aluminum nanocoating of optical quality. Measurement of the surface temperature of the studied substrate was carried out using a thermocouple, fixed at a distance of 1-2 $\mathrm{mm}$ from the contact line of the liquid drop. The temperature under the substrate was measured by three thermocouples of K-type. Thermocouples were placed radially at different distances from the centre of the disc and served to measure the temperature distribution along the substrate radius. The experimental setup was covered with transparent box made of PMMA which has dimensions of $1000 \times 800 \times 900 \mathrm{~mm}^{3}$. The relative humidity of air in the box during the experiments was 20$30 \%$.

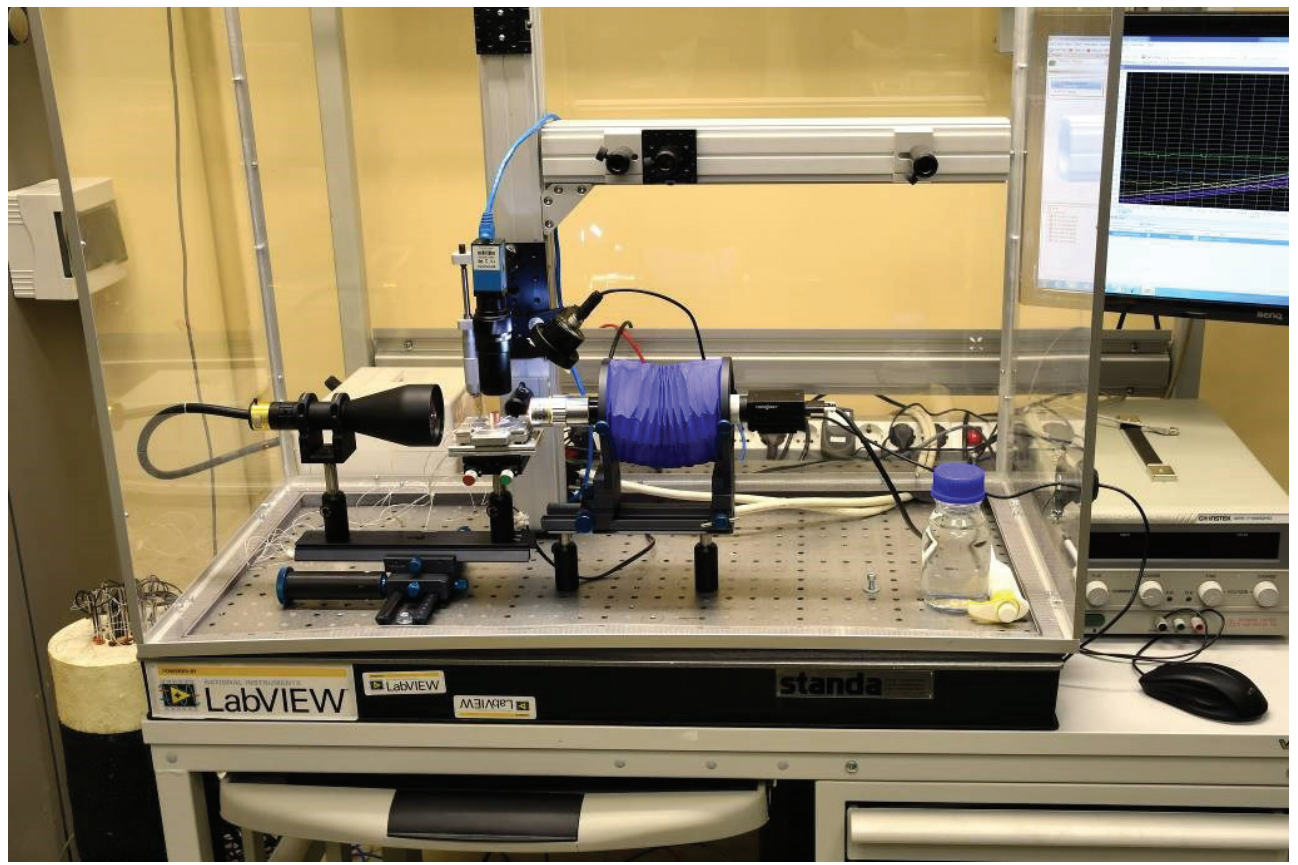

Fig. 1. Photography of experimental stand.

Two optical techniques were used. The first one is a shadow method, consisting of the halogen light source Edmunds Optics MI-150, connected by a fiberoptic lightguide with a system of lenses of Edmunds Optics Telecentric backlight illuminator. This system generates a plane-parallel light beam of $50 \mathrm{~mm}$ diameter at the output of one side of the work area. High-speed video camera FastVideo 500M with a microscope lens 10X Mitutoyo Plan Apo Infinity-Corrected Long WD, connected through the focusing bellow Novoflex Balpro TS, on the other side of the droplet. The spatial resolution of this system was $1 \mu \mathrm{m} /$ pixel, and the frequency of shooting was up to 500 frames per second. Using the shadow method we obtained shadow images of the liquid drop profile. The images were processed by the Drop Shape Analysis software of KRUSS company. Shadow photography was used to select the drop profile by the method of Young-Laplace. Further, the following geometric parameters of the liquid drop were determined: the base diameter, maximum diameter, height, volume, contact angles (right, left, middle), the surface area, and the basal area. 
The second optical technique consisted of a digital video camera Point Grey Grasshopper3 (4.1 Mpix) with a microscope lens 20X Mitutoyo Telecentric Objective. It was placed above the liquid drop and allowed visualizing the evaporation from the top and controlling the symmetry of the droplet. Spatial resolution of the system was $0.5 \mu \mathrm{m} / \mathrm{pixel}$, and the frequency of shooting was up to 90 frames per second. If the drop lost its symmetry, the experiment repeated.

\section{Results}

The experiment began with a preliminary preparation of the investigated substrate, which consisted in a thorough cleaning of the working surface. Then the substrate was placed in a reservoir with distilled water for a day. Thereafter, to remove residual water from the surface the substrate was flushed with clean compressed air designed for cleaning optical components. A liquid drop of pre-set size was placed on the surface, heated to the desired temperature, using a high-precision dispenser Lenpipet. The lens used in the shadow method was focused (depth of field was a few hundreds of $\mu \mathrm{m}$ ); and after that the periodic shooting was performed until complete evaporation of the liquid droplet. The upper camera was switched on simultaneously with the shadow method.

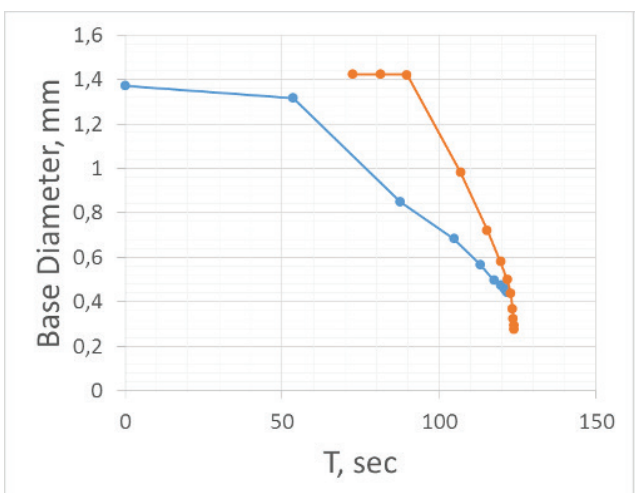

$\mathrm{a}$

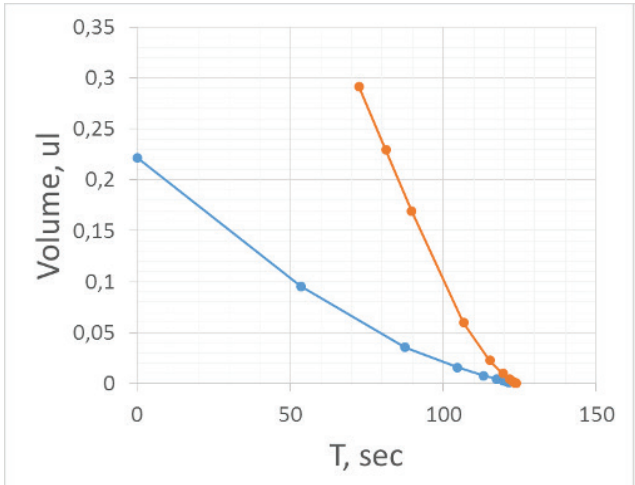

c

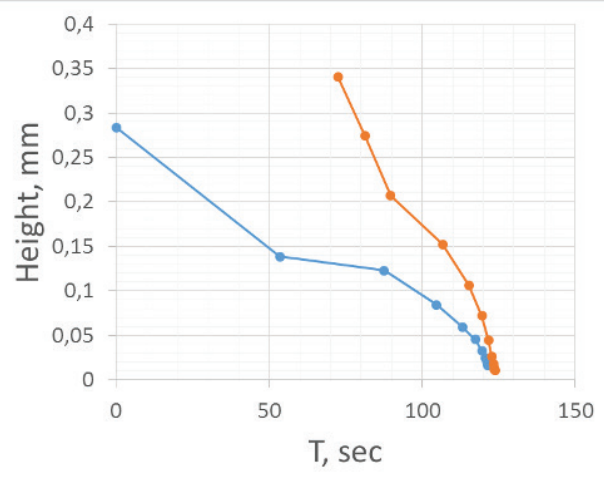

$\mathrm{b}$

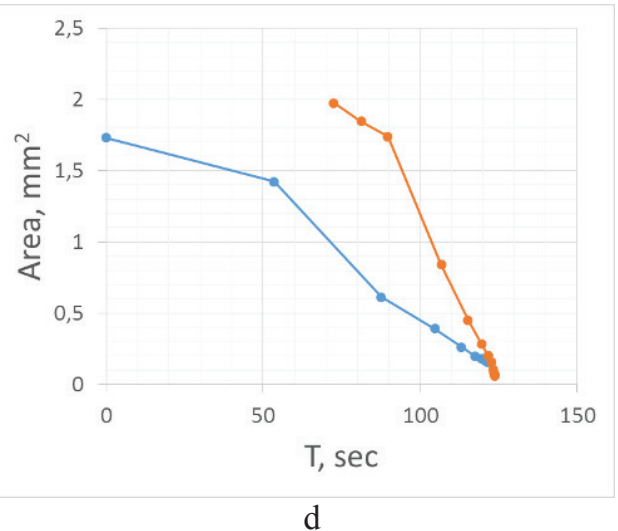

Fig. 2. Dependences of different parameters of droplet vs evaporation time. (a) - the diameter of droplet base, (b) - the height of droplet, (c) - the volume of droplet and (d) - the surface area of droplet. Blue lines and dots is data for substrate temperature $\mathrm{T}=30{ }^{\circ} \mathrm{C}$, orange $-\mathrm{T}=50{ }^{0} \mathrm{C}$. All data was synchronized for the moment of full droplet evaporation. 
Dependences of various parameters of a sessile liquid drop during evaporation vs time are shown in Fig.2. The obtained data are synchronized in time of complete evaporation of the droplet. Fig. 2 shows the difference between the evaporation of a drop of liquid on a substrate with an initial temperature of 30 (blue dots) and $50{ }^{\circ} \mathrm{C}$ (orange dots).

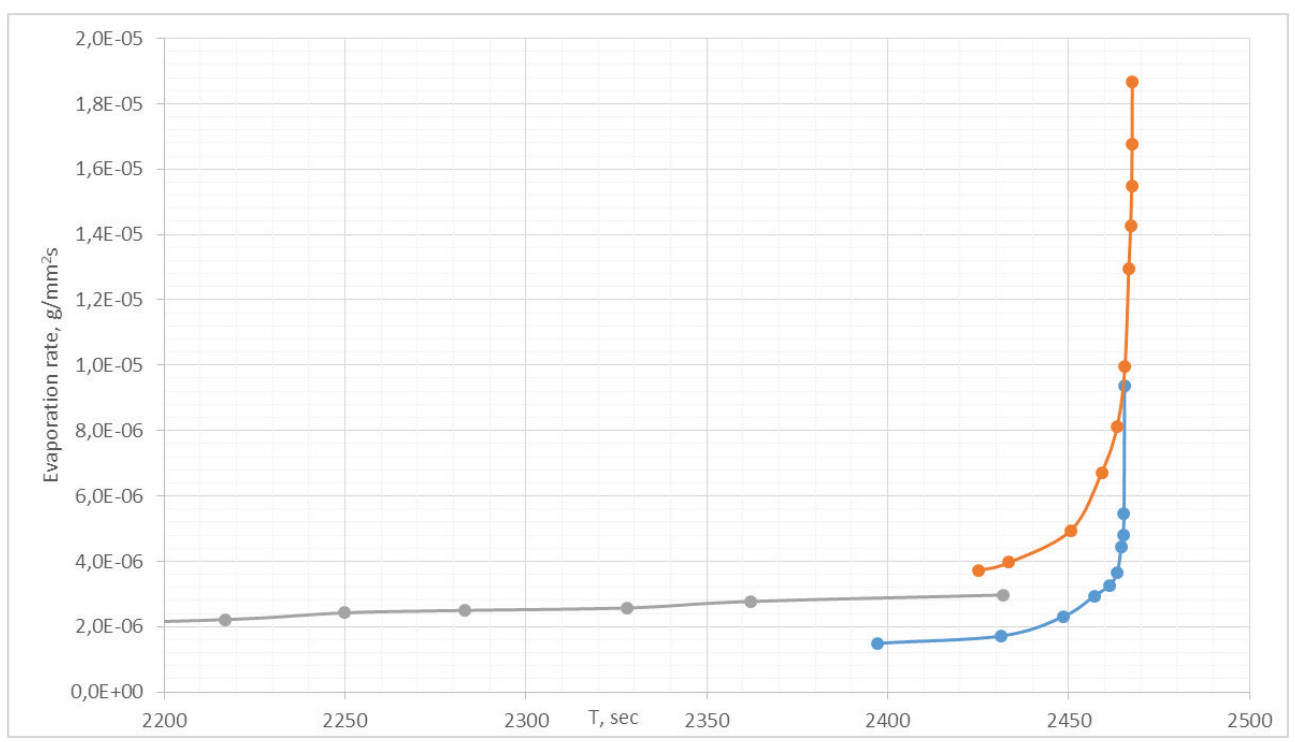

Fig. 3. The dependence of specific evaporation rate on time. Blue lines and dots - data obtained with the substrate temperature $\mathrm{T}=30^{\circ} \mathrm{C}$, the initial drop volume $\mathrm{V}_{0}=220 \mathrm{nl}$; orange $-\mathrm{T}=50^{\circ} \mathrm{C}, \mathrm{V}_{0}=290 \mathrm{nl}$; grey - data obtained in the work [2] at $\mathrm{T}=45^{\circ} \mathrm{C}, \mathrm{V}_{0}=95.16 \mu \mathrm{l}$.

The dependence of the specific rate of liquid evaporation on time at different initial surface temperature is shown in Fig. 3. The evaporation rate was calculated as a loss in the droplet mass per unit of droplet surface area per unit of time. All built-in data were synchronized to the time of complete evaporation of liquid droplets. The research has shown that the specific rate of evaporation increases with the decrease in droplet volume and at the last stage several times exceeds the initial value. The obtained results are in good agreement with the results of $[2,3]$, studying the evaporation of drops with initial volume of about 100 $\mu 1$.

This work was supported by the Russian Science Foundation (project 14-19-01755).

\section{References}

1. J. Kim, International Journal of Heat and Fluid Flow, 28, 753-767 (2006).

2. E.Ya. Gatapova, A.A. Semenov, D.V. Zaitsev, O.A. Kabov, Colloids and Surfaces A: Physicochemical and Engineering Aspects, 441, 776-785 (2014).

3. A.A. Semenov, D.V. Feoktistov, D.V. Zaitsev, G.V. Kuznetsov, and O.A. Kabov, Thermophysics and Aeromechanics, 22, 771- 774 (2015).

4. Kabov O.A. and Zaitsev D.V. Doklady Physics, 58, 292 (2013).

5. G.V. Kuznetsov, D.V. Feoktistov, E.G. Orlova, K.A. Batishcheva, Colloid Journal, 78, 335-339 (2016). 